\title{
Microstructural and Defect Characterization of Al-Si Alloy Using PFIB and EMPAD
}

\author{
Robert E. A. Williams ${ }^{1}$ \\ ${ }^{1 .}$ Center for Electron Microscopy and Analysis, The Ohio State University, Columbus, OH, USA.
}

Al-Si alloys have been widely utilized in automotive and aircraft applications, due in part, to their low density, good castability, favorable mechanical properties, abrasion resistance, good corrosion resistance, and low cost [1]. Since the inception of transmission electron microscopy (TEM), defects and microstructure have been characterized by conventional TEM, phase contrast imaging, which is supported by well developed theories and simulations [2]. As scanning transmission electron microscopy (STEM) has advanced with technological improvements, the concept of using STEM to image and characterize defects, has been shown to satisfy the dynamical theory of diffraction contrast and gained popularity [2-4].

More recently, the advent of technology that can improve how we characterize metallic alloys, specifically; aluminium alloys characterized using STEM has improved immensely. A partial list of these improvements could include high-brightness FEG sources, aberration correction, BF-STEM, dual electron energy loss spectroscopy (EELS), direct electron cameras, silicon drift detectors (SDD) and electron microscope pixel array detectors (EMPAD). The EMPAD is a direct electron detector with high dynamic range (HDR) and fast readout, allowing for digital capture of the full range of diffracted intensities while imaging/mapping, producing a 4D-STEM image [5]. Typical diffraction patterns range from intense signal within the transmitted beam to very weak signals found within some diffracted intensities. This large variation in signal requires a high dynamic range to capture in entirety. Following the collection of a 4D-STEM dataset, any type of virtual aperture, such as BF, DF, ADF, HAADF, DPC, iDPC or custom segmented, may be used to produce a resultant image. This capability has provided researchers with an unprecedented ability to capture almost all of the desired diffraction information within a single EMPAD image.

Critical to analytical STEM characterization for aluminium alloys is the ability to produce a thin, damage free specimen. Historically, this was performed using electropolishing for acceptable aluminium alloys, and was improved with the introduction of the $\mathrm{Ga}^{+}$focused ion beam (FIB). The $\mathrm{Ga}^{+}$ FIB allowed for STEM lamellae preparation of all aluminium alloys, however, $\mathrm{Ga}^{+}$wets aluminium grain boundaries and interfaces leaving behind significant amounts of $\mathrm{Ga}^{+}$and in some cases creating second phases [5]. This interferes with analytical STEM characterization and is prohibitive when identifying minor alloying additions through xray energy dispersive spectroscopy (XEDS). One possible solution to these barriers is a $\mathrm{Xe}^{+}$plasma focused ion beam (PFIB), which has shown great promise for improving STEM lamellas extracted from aluminium alloys [6,7].

The work to be presented will discuss the improvements in STEM lamellae quality as a result of PFIB sample preparation, as compared to $\mathrm{Ga}^{+}$FIB. By removing the residual $\mathrm{Ga}^{+}$and reducing the surface damage layer, analytical characterization of microstructural features such as dislocations, twins, small scale precipitates, and analytical XEDS was improved and in some case enabled by the PFIB preparation. Complementing the characterization analysis of the ion induced damage layers, the 4D EMPAD imaging allowed for rapid $\mathrm{G} \bullet b$ analysis of large areas through virtual apertures and post 
processing as shown in Fig 1. The use of the EMPAD for diffraction contrast imaging of defects will also be discussed.

\section{References:}

[1] X Sha et al., Materials 11 (2018), p. 1151.

[2] Y Zhu et al., Ultramicroscopy 193 (2018), p. 12.

[3] PJ Phillips et al., Ultramicroscopy 111 (2011), p. 1483.

[4] PG Callahan et al., Ultramicroscopy 186 (2018), p. 49.

[5] M Tate et al., Microscopy and Microanalysis 22 (2016). p. 237.

[6] K Unocic, MJ Mills, and GS Daehn, Journal of Microscopy 240 (2010), p. 227.

[7] K Fisher and E Marquis, Microscopy and Microanalysis 22 (2016), p. 692.

[8] A Ernst, M Wei, MA Aindow, Microscopy and Microanalysis 23 (2017), p. 288.

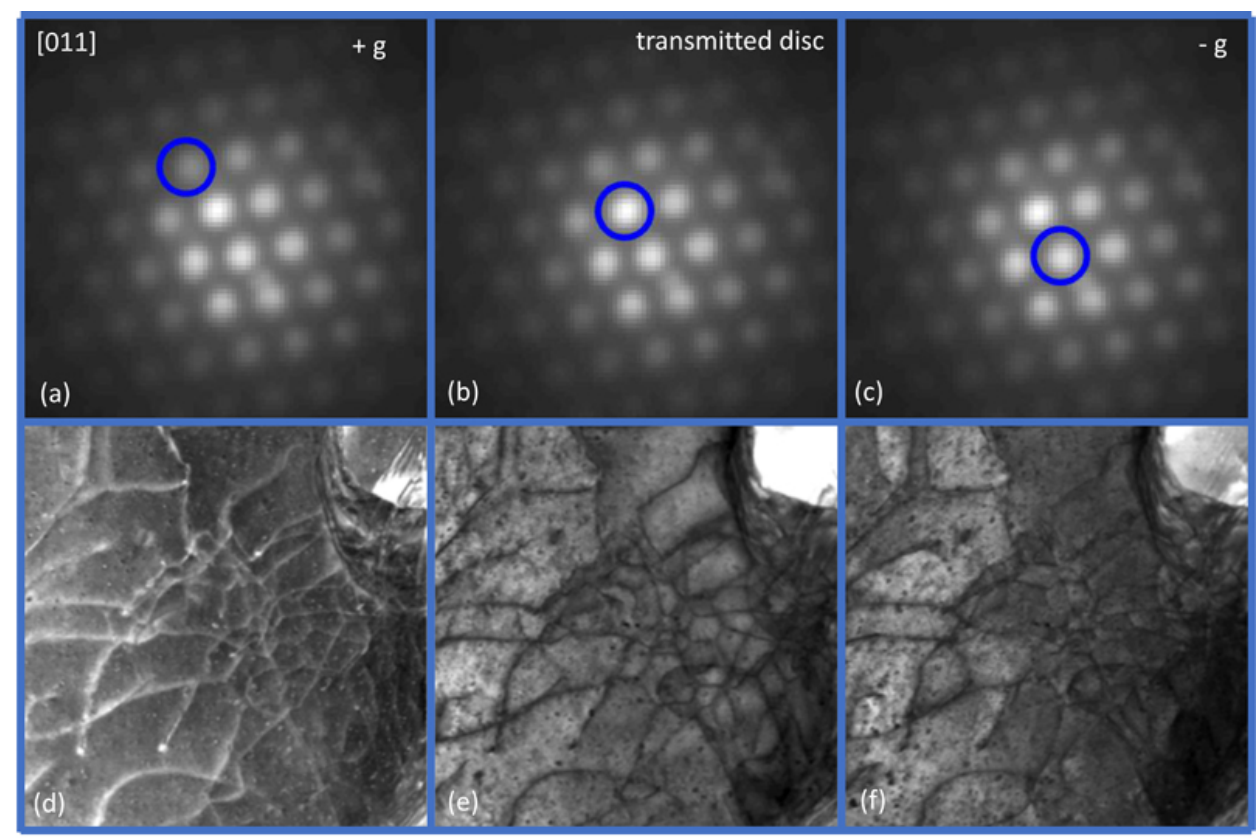

Figure 1. (a-c) Raw EMPAD images of [100]Al. Blue circles indicate virtual aperture used to create. (d-f) Diffraction contract images extracted from raw EMPAD image. 